(Aus dem physiologischen Institut zu Königsberg i. Pr.)

\title{
Beiträge
}

\section{zur Lehre von den Haut- und Secretionsströmen.}

Nach Versuchen des Herrn Dr. Wartan v. Wartanoff aus St. Petersburg (Sommer 1893) und der Herren cand. med. Kurt Schmarsow (Winter 1893-94) und cand. med. Paul Junins (Frühjahr 1894).

Von

\section{Hermann.}

1. Die Hautströme des Laubfrosches (Hyla arborea).

Herr Junius hat im Frïhjahr dieses Jahres die Hautströme des Laubfrosches und deren Schwankungen einer Untersuchung unterzogen, deren Ergebnisse hier kurz mitgetheilt werden sollen.

Die Haut zeigt überall einen einsteigenden Strom, dessen electromotorische Kraft bis etwa 0,015 Volt gefunden wurde, also geringer ist als bei Rana temporaria und esculenta. Die Färbung des untersuchten Hautstïckes ist auch hier ohne deutlichen Einfluss auf den Strom. Bei Aufbewahren ausgeschnittener Hautstiicke in de: feuchten Kammer sinkt die Kraft sehr allmählich auf Null. Reizversuche scheinen dieses langsame Sinken zu befördern.

Zur Untersuchung des Einflusses der Nervenreizung wurde durchgehends die Unterschenkelhaut benutzt, da ein Rïckenhautpräparat, wie ich es bei Fröschen vielfach verwendet habe, sich hier wegen des Nervenverlaufes nicht anfertigen lässt. Am Knie war der Stumpf der Knochen und der Muskeln, sowie der Nervus ischiadicus erhalten. Reizung des Ischiadicus bewirkte stets eine kräftige positive Schwankung, d. h. einen einsteigenden Secretionsstrom. Schon bei erheblichem Rollenabstand $(150 \mathrm{~mm})$ beträgt derselbe 400 Sealentheile und mehr, um bei Wiederholungen allmählich bis etwa auf die Hälfte und mehr abzunehmen. Stets ist eine deutliche Latenzzeit vorhanden. In manchen Fällen geht ein kleiner negativer Vorsehlag von 5-10 Scalen- 
theilen voraus; in der Mehrzahl der Fälle fehlt derselbe, und die Schwankung ist ausschliesslich positiv.

Mit der Reizung ist deutliche Secretion von schwach saurer Reaction verbunden.

Das galvanische Verhalten ist also genau dasselbe, wie ich es vor 16 Jahren am Rückenpräparat des Frosches gefunden habe.

Um zu sehen, ob der gewöhnliche Aufenthalt der Thiere im Trocknen einen Antheil an dem Verhalten hat, wurden einzelne Laubfrösche vor dem Versuch einen Tag lang mit Ausschluss des Kopfes unter Wasser versenkt. Das Verhalten der Haut in der Ruhe und bei Nervenreizung erlitt aber dadureh keine Veränderung.

Die Versuche wurden ausserdem nach einer von mir angegebenen, jetzt etwas modificirten Methode ${ }^{1}$ ) am ganzen Thiere untersucht. Der Laubfrosch wurde curarisirt, von zwei beliebigen Hautstellen, deren eine vorher mit gesättigter Kochsalzlösung geätzt war, zum Galvanometer abgeleitet, der von der ungeätzten zur geätzten Stelle gerichtete Strom compensirt, und nun das Rückenmark tetanisirt. Auch hier ergab sich stets ein einsteigender Secretionsstrom der ungeätzten Stelle, welchem nur zuweilen ein aussteigender Vorschlag vorangeht.

2. Das Verbalten des $01 \mathrm{mes}$ (Proteus anguineus).

An zwei aus dem Berliner Aquarium bezogenen Olmen (ausgewachsen, ein Männchen und ein Weibchen) hat Herr Junius folgende Versuche angestellt.

Die Haut lässt sich nirgends in grösserer Ausdehnung vom Muskelkörper genügend abtrennen. Die Ruheströme konnten daher nur in situ zwischen einer mit gesättigter Kochsalzlösung geätzten und einer ungeätzten Stelle untersucht werden. Sie sind stets einsteigend, die electromotorische Kraft betrug an Rumpfe im Maximum 0,0112 Volt. An den Extremitäten wurde sie beträchtlich höher, nämlich zwischen 0,033 und 0,056 Volt gefunden.

Die Erregung wurde in der oben angegebenen Art durch Tetanisirung des Ruckenmarks am curarisirten Thiere vorgenom.

1) Dies Archiv Bd.17. S. 298. Damals wurde die indifferente Ableitung auf einen Schenkel, dessen Nerv durchschnitten war, jetzt auf eine geätzte Hautstelle applicirt; beides kommt auf dasselbe hinaus. 
men. An allen untersnchten Stellen überwogen bei weitem die einsteigenden Wirkungen, welche jedoch nie tuber 100 Scalentheile hinausgingen. Nur in einzelnen Fällen wurde ein aussteigender Vorschlag beobachtet. Die einsteigenden Wirkungen gehen oft noch während der Reizung auf Null zurück, zuerst schnell, dann langsamer; einige Male gingen sie ïber den Nullpunkt bis etwa 50 sc. nach der negativen Seite hinaus, nachdem um Null kleine Oscillationen stattgefunden hatten. Jede Reizung hinterlässt in solchen Fällen den Ruhestrom bleibend geschwächt. An den Extremitäten ging merkwürdiger Weise die positive Wirkung weder während der Reizung noch nach derselben zurïck, so dass der Ruhestrom dauernd verstärkt blieb, und durch jede folgende Reizung noch weiter verstärkt wurde.

Die Versuche werden fortgesetzt.

3. Das Verbaltendes Axolotl (Amblystomamexic an u $\mathrm{m}$ ).

4 aus dem Berliner Aquarium bezogene kräftige Thiere dienten zu den von Herrn Junius angestellten Untersuchungen. Auch hier lässt sich die Haut am grössten Theil des Körpers nicht isolirt untersuchen, sondern es muss von einer geätzten und einer unversehrten Hautstelle abgeleitet werden; der Strom ist überall einsteigend, die Kraft etwa 0,005-0,010 Volt. An der Schwanzhaut konnte der mässige einsteigende Ruhestrom auch bei $\mathrm{Ab}$. leitung von Aussen- und Innenfläche isolirter Stïcke constatirt werden.

Die Erregung erfolgte auch hier vom Rückenmark aus am curarisirten Thiere. Bei hinreichend starkem Tetanisiren entsteht in der Regel schwache $\mathrm{neg}$ a ti v e Schwankung des Ruhestroms, also a usteig e nde Wirkung, zuweilen mit kleinem $p$ os it iv e m (einsteigendem) Vorschlag. In einem Falle war die primäre einsteigende Wirkung die überwiegende, und die nachfolgende aussteigende schwach.

Das letztere Verhalten wurde an den Hinterbeinen auch bei Reizung des Ischiadicus beobachtet.

Die Versuche an den genannten und an anderen Amphibien werden von Herrn Junius fortgesetzt. Derselbe hat auch über die Wirkungen directer Hautreizungen Versuche angestellt, welche später mitgetheilt werden sollen. 
4. Ueber Hautströme und reflectoriscbe Hauterregungen beim Warmblüter.

Im Sommer 1893 hat Herr Dr. v. Wartanoff auf meine Veranlassung eine grössere Versuchsreihe über Hautströme bei Katzen angestellt ${ }^{1}$ ). Die Thiere waren curarisirt und unter künstlicher Respiration.

Der Ruhestrom der Haut, welcher an nackten Hautstellen untersucht wurde, ist stets einsteigend. Dies konnte sowohl an ausgeschnittenen Hautstïcken, als auch in situ durch Ableitung von einer unversehrten Hautstelle und der unversehrten Oberfläche blosgelegter Muskeln nachgewiesen werden. Abtragung der Epithelschicht schwächt den Strom sehr beträchtlich, was auf eine Betheiligung des Epithels an der electromotorischen Kraft hindeutet, da die Catis mit den Schweissdruisen unverletzt blieb.

Pilocarpininjection in eine Pfote bewirkt bei symmetrischer Ableitung von beiden Pfoten, welche für sich schwache Differenzströme von wechselnder Richtung ergiebt, stets einen kräftigen Strom von der Injectionsseite zur anderen, verstärkt also den einsteigenden Hautstrom. Dasselbe lässt sich auch dadurch zeigen, dass man in einer Pfote durch eine elastische Umschnürung die Circulation hemmt, und nun Pilocarpin in die Jugularvene injicirt: allmählich entsteht ein Strom von der nicht unterbundenen zur unterbundenen Pfote, welcher nach dem Abnehmen der Ligatur allmählich verschwindet. In einem Falle blieben diese Erfolge aus, in einem anderen kehrte sich der durch Pilocarpin bewirkte Differenzstrom nach längerer Zeit um.

Atropininjectionen haben auf den Ruhestrom der Haut anscheinend keine irgend erhebliche oder constante Wirkung.

Reizung des peripherischen Endes eines Ischiadicus bewirkt, wie ich schon mit Luchsinger fand, stets einen von der Pfote der gereizten za derjenigen der ungereizten Seite gerichteten Strom, welcher am atropinisirten Thiere vollständig ansbleibt. Diese Wirkung ist auch dann vorhanden, wenn die Epidermis auf der gereizten Seite möglichst vollständig abgetragen ist, ist also jedenfalls

1) Eine sehr ausgedehnte von Herrn Dr. Wendt aus St. Petersburg im Jahre 1887 im hiesigen Institut ausgeführte Untersuchung über Hautund Secretionsströme am Menschen wurde leider nicht zum Abschluss und zur Publication gebracht. 
ganz oder theilweise Wirkung der erregten Driisen. Wird die schichtweise Abtragung bis zum Eintritt einer Blutung fortgesetzt, so werden die Wirkungen bis zum Verschwinden geschwächt.

Reizung cines centralen Ischiadicusendes bewirkt jedesmal einen Strom von der ungereizten zur gereizten Seite, deren Drüsen durch die Nervendurchschneidung vom Centralorgan abgetrennt sind. Dieselbe Wirkung hat auch die Reizung des centralen Cruralisendes; der Strom geht von derjenigen Pfote, deren Ischiadicus nicht durchschnitten ist, zur gelähmten Seite. Beide Nerven rufen also einen bilateralen einsteigenden Secretionsstrom reflectorisch hervor. Das Latenzstadium dieser reflectorischen Wirkungen ist deutlich länger als dasjenige der directen.

Befindet sich das ganze Thier in einem Wärmekasten, und wird nach Durchschneidung eines Ischiadicus von beiden Pfoten abgeleitet, so bewirkt Erhitzung stets einen Strom von der unversehrten zur gelähmten Seite. Hitze bewirkt also durch centrale oder reflectorische Erregung einen einsteigenden Secretionsstrom.

5. B emerkungen zurTheorieder Haut-und Secretionsström e.

Auf Grund meiner Versuche, sowie derjenigen meiner Mitarbeiter Luchsinger, Bach, Oehler und v. Gendre ${ }^{1}$ ) habe ich die frïher ganz unvermittelt dastehende Erscheinung der von $d u$ B o is-Reymond entdeckten Hautströme und ihrer Erregungsveränderungen auf dasselbe Grundprincip zurückgeführt, auf welches ich alle electromotorisehen Wirkungen der Maskeln and Nerven zurückführen konnte, nämlich auf die Negativität des sich apobiotisch ${ }^{2}$ ) alterirenden Protoplamas gegen das nicht oder weniger alterirte Continuum. Dieser zuerst vor 12 Jahren gegebenen Ableitung hat sich neuerdings Biedermann vollkommen angeschlossen ${ }^{3}$ ). Nur in zwei weniger wesentlichen Pankten weicht dieser Autor von mir ab.

1) Vgl. dies Archiv Bd. 17. S. 291, 310, Bd. 18. S. 460, 471, Bd. 22. S. 30, Bd. 27. S. 280 , Bd. 34. S. 422.

2) Als "apobiotische Alteration" des Protoplasma bezeichne ich schon seit langer Zeit in meinen Vorlesungen alle diejenigen Veränderungen, welche die protoplasmatische Lebensenergie temporär oder bleibend vermindern, also Erregung, Absterben, Metamorphose zu Schleim, Horn oder dergl.

3) Dies Archiv Bd. 54. S. 209. Da in dieser Abhandlung die Abwei- 
Erstens will sich Biederman n meiner Aufstellung, dass nicht bloss die Drüsen, sondern auch das Epithel der Haut an dem einsteigenden Ruhestrom einen Antheil hat, nicht anschliessen. Er bestätigt zwar vollständig die von mir zuerst, gegenüber den negativen Ergebnissen d u Bois - Re y mond's, nachgewiesenen einsteigenden Ströme der drüsenlosen Fis chh a ut (Bd.27. S. 281), macht aber den, wie er sagt, "nabeliegenden Einwand, " dass derselbe von den einzelligen Drüsen derselben, nämlich den Becherzellen herribhrt. Diesen Umstand habe ich keineswegs, wie es nach Biedermann's Darstellung scheinen könnte, und wie soeben E. W. Reid sogar bestimmt behauptet ${ }^{1}$ ), übersehen, vielmehr die schleimbilden den Zellen der Fischbaut ganz ausdricklich hervorgehoben und mit den Becherzellen des Darmes auf gleiche Linie gestellt (Bd. 27. S. 285). Diese Zellen sind doch aber nun einmal Epithelien, und meine Behauptung ging eben dahin, dass auch das freie Epithel, und nicht bloss dasjenige der Drüsen, einen Strom verursacht ${ }^{2}$ ). Dass aber n a r schleimbildende, und nicht auch verhornende Epithelien diese Wirkung haben, hat weder Biedermann, der nur von "vielleicht zu erhebenden Einwänden" spricht, ohne dieselben anzuführen $^{3}$ ), noch irgend ein Anderer bewiesen. Auch die Verhornung ist eine orientirt von aussen nach innen fortschreitende apobiotische Veränderung, welche höchstwahrscheinlich auch innerhalb der Einzelzellen Demarcationsflächen schafft, und es wäre fast wunderbar, wenn sie nicht ebenfalls zu einer einsteigenden electromotorischen Kraft Anlass gäbe. Ich habe aber weiter eine hervorragende Betheiligung der Froschhautdriisen an dem sehr kräftigen einsteigenden Ruhestrom der Haut als unwahrscheinlich bezeichnet,

chungen stärker betont sind, als die weit überwiegenden Bestätigungen, werde ich auch hier die ersteren besonders zu prüfen haben.

1) Journ. of physiol. Bd. 16. S. 360. Grade dieser Autor bringt neue Beweise für die Wirksamkeit driisenloser Epithelien.

2) Es kommt noch hinzu, dass Biedermann nur am Aal, ich aber an den verschiedensten, auch an kaum secernirenden Fischen Versuche angestellt habe.

3) Wenn diese Einwände etwa darin liegen sollen, dass an der Froschhaut eine Verhornung nicht nachweisbar sein soll (S. 257), so bildet wohl schon die Abstossung der obersten Lagen einen genügenden Nachweis, und das Princip wäre nicht einmal an das Auftreten eigentlichen Keratins als metamorphotisches Product gebunden. 
weil diese kugligen Gebilde für gewöhnlich durch eine Versehlusszelle von der Oberfläche so abgeschlossen sind, dass gar nicht abzusehen ist, wie die electromotorischen Kräfte ihrer Zellen nach aussen zur Ableitung. kommen sollen. Die Meinung Bie dermann's (S. 272), dass die capillare Secretschicht, welehe die Haut bedeckt, und mit dem Secretinhalt der Drüsen in unmittelbarem Contact steht, genijgend wirksam rom Drïseninhalt ableitet, bin ich weit entfernt zu theilen. Man braucht nur die Versehlusszellen in situ zu betrachten, um sich zu uberzeugen, dass diese Ableitung erst im Augenblick des Secretaustrittes ergiebig werden kann. Und hierin sehe ich eine sehr starke Bestätigung meiner Meinung, dass auch der Verhornungsprocess des Hautepithels einen wesentlichen Antheil am Ruhestrom bat, ja denselben vielleicht überwiegend bedingt. Einen weiteren Beweis habe ich mit Bach \& Oehler dadurch geführt, dass Sublimatbäder den Ruhestrom der Froschhaut beseitigen können, ohne die Drtisen zu tödten, deren Secretionsstrom bestehen bleibt ${ }^{1}$ ). Die soeben erschienene, bereits erwähnte Árbeit von Reid scheint meine Behauptung, dass driisenlose verhornende Epithelien einen einsteigenden Strom besitzen, auch für den Warmblüter zu bestätigen. Nach wie vor halte ich auch Engelmann's Meinung, dass Flimmerzellen einen einsteigenden Strom geben, für höchst wahrscheinlich, obwobl ich Biedermann's Einwand (S. 245, 247), dass auch flimmerlose Schleimhäute denselben Strom besitzen, bereits selbst gemacht habe ${ }^{2}$ ).

Dass ich aber, wie Biedermann sagt (S. 212), zwischen Rube- und Secretionsstrom "streng" unterscheide, and zwischen Drüsen- und Epithelstrom einen "so zu sagen principiellen Unterschied" mache (S. 214), der "in keiner Weise gerechtfertigt sei", ist doch grade das Gegentheil meiner klar ausgesprochenen Stellung, welche ganz ausdrücklich Ruhe- und Secretionsströme, Epithelund Drüsenströme auf eine gemeinsame Ursache, nämlich auf orientirte Alteration des Zellprotoplasma zurïckführt ${ }^{3}$ ). Was Biederma.nn als Ergebniss seiner Arbeit ausspricht, dass alle diese Ströme "Zellströme" sind, dass schon der "Ruhestrom" als „Secretionsstrom" anzusprechen ist, und beide von denselben Elementen aus-

1) Dies Archiv Bd. 22. S. 33.

2) Dies Archiv Bd. 27. S. 285. Anm. 2.

3) Schon in meiner ersten Arbeit von 1877 habe ich Ruhe und Secretionsströme der Haut identificirt (dies Archiv Bd. 17. S. 302 f.). 
gehen (S. 275), dies zuerst erkannt zu haben, muss ich fïr mich in Anspruch nebmen. Ich war es, der den Drüsencpithelien einen beständigen Secretionsstrom zuschrieb, welcher nur weit weniger günstig zur Ableitung kommt, als der gleichsinnige und von analoger Ursache herrïhrende des freien Epithels. Wenn ich dabei die Antheile der freien und der Drisenepithelien, and zwar im sog. Ruhezustand und im thätigen, richtiger thätigeren Zustand experimentell zu sondern und isolirt darzustellen versuchte, so heisst das nun und nimmermehr einen principiellen Uuterschied machen.

Die zweite, mehr thatsächliche Differenz betrifft den einsteigenden Secretionsstrom, den ich und meine Mitarbeiter sowohl an der Haut wie an der Zungenschleimhaut als iuberwiegenden Erfolg der Nervenreizung beobachtet baben, während Biederman ihn für etwas Abnormes erklärt, und unter "normalen" Verbältnissen nur aussteigende (negative) Wirkungen erhalten hat.

Ich halte dagegen nach wie vor das Ergebniss unserer sehr umfangreichen und über viele Jahre sich erstreckenden Versucbe für ebenso „normal,“ wie dasjenige Biedermann's. A n der Froschbaut ỉberwiegt, wie ich ${ }^{1}$, und später Bach $\& 0$ eble ${ }^{2}$ ) fanden, und Biedermann lediglich bestätigt, um so mebrdereinsteigende Secretionsstrom, jeschwächer der Rubestrom. Die aussteigenden Wirkungen mischen sich dann entweder gar nicht, oder als Vor- oder Nachschläge ein, während bei starkem Ruhestrom rein aussteigende Wirkungen auftreten. Welches Recht aber hat man, den starken Rubestrom als die Norm, und den schwachen als abnorm zu bezeichnen? Biedermann scheint mir nicht genügend erwogen zu haben, dass ich monatelang in Zürich nur rein einsteigende Secretionsströme beobachtet habe ${ }^{3}$ ), an völlig normalen, frisch eingefangenen Fröschen, und dass die Nervenreizung stets reichliche Secretion lieferte, dass B y liss \& Bradford

1) Dies Archiv Bd. 17. S. 308, Bd. 18. S. 472.

2) Dies Archiv Bd. 22. S. 33.

3) Dass nach Biedermann das von mir hauptsächlich angewandte, von Muskeleinmischung absolut freie Rückenhautpräparat weniger schonend sein soll als das Schenkelpräparat (S. 259), kann ich ebensowenig zugeben, wie die analoge Bemerkung gegen die abgezogene Aalhaut (S. 256), an welcher ich doch die von Biedermann bestätigten Fischhautströme zuerst nachgewiesen habe. 
während des grösseren Theiles des Jahres ganz dasselbe fanden ${ }^{1}$ ), vor Allem aber, dass, wie ich zuerst mit $\mathrm{Lu} \mathrm{ch}$ s inger fand, der Warmblüter und der Me n s ch (d u Bois'schen Willkürversuch etc.) überhaupt durchweg $n u r$ einsteigende Secretionsströme liefern ${ }^{2}$ ), was alle Beobachter bestätigt haben ${ }^{3}$ ). Es kommt jetzt das von $J$ u n i u beobachtete gleiche Verhalten des Laubfrosches and des Olmes hinzu (s.oben). Was die Froschzunge betrifft, so habe ich mit L u c h sing er beobachtet ${ }^{4}$ ), dass der auf Reizung des Hypoglossus (schwächer auch bei derjenigen des Glossopharyngeus) anftretende einsteigende Secretionsstrom die Grundwirkung ist, welche aber vorübergehend durch einen aussteigenden Strom übercompensirt wird, so dass die Wirkung dreiphasisch wird; B i e d e r man $\mathrm{n}$ sieht das letztere Verhalten wiederum nur bei geschwächtem Ruhestrom. Auch hier finde ich nicht den leisesten Grund, unser Ergebniss, welches mit völlig tadelfreier Methode gewonnen ist, als weniger normal zu betrachten. Wir haben auch hier stets die Secretion festgestellt, und haben die electromotorische Kraft des Ruhestroms ausdrücklich vermerkt, was ich bei Biedermann vermisse, wir haben endlich angegeben, dass wir uns auf die schwächeren Ströme beschränkt haben, und ausserdem der Nerv durch einen aufgelegten Schutznerven eine Nebenschliessung hatte; dass starke Reize die aussteigende Wirkung begünstigen, ist, wenigstens für das Ruickenpräparat der Kröte ${ }^{5}$ ), von Herrn Ju nius wiederholt beobachtet worden. Ferner hat auch Biedermann (S. 251, 252) wiederholt an Schleimhäuten bei schwachen Reizungen einsteigende, bei starken aussteigende Wirkungen beobachtet, ebenso Reid \& Tolputt6).

Ich bin weit entfernt, Bi edermann's Resultate an der Froschhaut, welche mit denjenigen von Roeber und Engelmann iibereinstimmen, als weniger massgebend anzusehen. Auch mir, sowie den Herren Schmarsow und Junius, ist hier in Königsberg der rein einsteigende Secretionsstrom bei weitem nicht so regelmässig

1) Journ. of physiol. Bd. 7. S. 217.

2) Dies Archiv Bd. 17. S. 310.

3) U. A. Bubnoff, Arch. f. microsc. Anat. Bd. 20. S. 108; Tarchanoff, dies Archiv Bd. 46. S. 46; Wartanoff, s. oben.

4) Dies Archiv Bd. 18. S. 467.

5) Vgl. dies Archiv Bd. 17. S. $299 \mathrm{f}$.

6) Journ. of physiol. Bd. 16. S. 215. 
vorgekommen, als in Zitrich. Schon Ostern 1878 habe ich in Breslau, als ich den Herren $\mathrm{H}$ e ide $\mathrm{n} \mathrm{hain} \mathrm{und} \mathrm{Gr}$ ü $\mathrm{tz}$ er an einer Esculenta den einsteigenden Strom am Rückenpräparat demonstriren wollte, der unmittelbar vorher in Zürich so unendlich oft von mir beobachtet war, zu meiner Ueberraschung nur aussteigende Wirkung erhalten. Herr Schmarsow beobachtete in Königsberg im vorigen Wintersemester rein aussteigende Wirkungen in 66 , rein einsteigende in 12, und doppelsinnige in 22 Procent der Fälle. Trotz immer wiederholter Bemühungen kann ich den Grund dieser Abweichung nicht angeben; da er sich auch auf die Zunge zu erstrecken scheint (s. oben), so halte ich es für das Wahrscheinlichste, dass die Züricher und die mittel- und norddeutschen Frösche eine kleine Verschiedenheit in dieser Richtung besitzen, welche vielleicht mit der Beschaffenheit des Wassers zusammenhängen mag; diese Verschiedenheit braucht nur sehr klein zu sein, da auch hier Frösche mit rein einsteigenden Secretionsströmen keineswegs sehr selten sind. Die feuchtere oder trocknere Aufbewahrung hat, wie ich sorgfältig constatirt habe, keinen Einfluss, ebensowenig kann ich mich nach meinen Erfahrungen der Meinung von Bayliss \& Brad ford (a. a. 0.), dass die Jahreszeit den Unterschied bedinge, anschliessen. Auch Geschlecht, Paarungszeit u. dgl. fand ich ohne Einfluss. Nur das muss ich mit allem Nachdruck festhalten, dass das Auftreten rein einsteigender Wirkungen an ebenso "normalen" Thieren vorkommt, wie dasjenige gemischter oder rein aussteigender.

Dass jedoch von den beiden mit einander kämpfenden Wirkungen die einsteigende als eigentlicher Secretionsstrom zu bezeichnen ist, scheint mir zweifellos, weil sie erstens bei vielen $0 b-$ jecten, u. A. beim Laubfrosch und beim Warmblüter, die einzige, und zweitens stets mit deutlicher Secretion verbunden ist. Auch ist diese Wirkung, was freilich für die Thatsachen wenig besagen will, aus dem von mir aufgestellten Princip, welchem sich auch Biedermann anschliesst, leicht verständlich, während die aussteigenden Wirkungen sich vorläufig einem klaren Verständniss entziehen.

Zur Erklärung dieser aussteigenden Wirkungen habe ich im Laufe der Zeit auf drei verschiedene Möglichkeiten hingewiesen: 1. es werden neben den secretorischen auch antagonistische, hemmende Fasern erregt ${ }^{1}$ ), welche den beständigen Zell-

1) Bd. 17. S. 303. 
process herabsetzen und dadurch eine negative Schwankung des Ruhestroms herbeiführen; 2. Drïsen, welche alkalisches und solche welche saures Secret liefern, könnten entgegengesetzte Secretionsströme haben ${ }^{1}$ ); 3. der Umstand, dass die Drüsencomponente des einsteigenden Stroms der Froschbaut neben der Epithelcomponente in der Rube wegen des Versehlusses der Drüsen kaum oder gar nicht zur Geltung kommt, dagegen stark, sobald Secret ausgepresst wird, kann sowohl positive wie negative Schwankungen bei der Reizang erklären, sobald beide Componenten an electromotorischer Kraft verschieden sind, oder es bei der Nervenreizung werden ${ }^{2}$ ). Die Einwände, welche Bied e r m a n $n$ gegen diese letztere ganz unanfechtbare Aufstellung erhebt, sind bedeutungslos; dass sie für die Zungendrüsen nicht passt, für welche ich sie gar nicht aufgestellt habe, will Nichts für die Haut sagen; und dass die capillare Secretschicht, welche die Haut bedeckt, eine erheblich wirksame Ableitung vom Drüsenlumen darstellen soll, wird wie schon bemerkt Niemand zugeben, der die Verschlusszellen der Drüsen betrachtet.

Biedermann's eigene Erklärung der aussteigenden Wirkungen ist ebenfalls nur eine Vermuthung, und was ich an derselben zutreffend finde, ist wie sich zeigen wird nichts Anderes als was ich oben sub 1. angefuhht habe. Er schliesst sich meiner Ansicht an, dass die einsteigenden Ruhe- und Secretionsströme von der secretorischen schleimigen (nach mir auch hornigen) Alteration der Aussenzonen der Zellen herrühren; die aussteigenden Ströme aber sollen von einer entgegengesetzten Zellenveränderung (meiner synthetischen, $\mathrm{He}$ r in g's assimilativen oder aufsteigenden) bewirkt werden ${ }^{3}$ ).

Hier bleibt erstens unklar, wie dieser entgegengesetzte Zellprocess electromotorisch wirken soll, da noch eine Annahme über Orientirung desselben hinzukommen müsste. Damit die Wirkung aussteigend sei, müsste die synthetische oder aufsteigende Veränderung ebenfalls primär die Aussenzonen ergreifen, was mir weniger wahrscheinlich vorkommt, als dass sie im Gegentheil von der gefässhaltigen Matrix ausgeht; dann aber würde sie wiederum einen einsteigenden Strom bewirken müssen.

Weiter aber kann doch wohl kaum angenommen werden, dass es diesel ben Nervenfasern sind, deren Erregung absteigende oder
1) Bd. 17. S. 309 .
2) Bd. 27. S. 286 .

3) Biedermann macht sich zwar S. 274 gegen diese Aufstellung Einwände, kommt aber, soviel ich sehe, S. $275 \mathrm{f}$. wieder auf dieselbe zurück. 
aufsteigende Veränderung macht, je nachdem vorber der secretorische Process schwach oder stark war, mit anderen Worten, dass die Nervenerregung die Zelle jedesmal gleichsam auf einen mittleren Zustand einstellt. Wie sollte da uberhaupt das Nervensystem kräftige Secretion einleiten können? Auch Biedermann wird nichts Anderes übrig bleiben, als zweierlei antagonistische Fasern anzunehmen, secretorische und hemmende. Das wäre also meine zuerst aufgestellte Annabme. Man braucht dann aber, ja man darf uiberhaupt - da die Hypothesen nie weiter gehen sollen als die Thatsachen nöthigen - antagonistische Zellprocesse gar nicht annehmen. Die beiden Nervenfasergattungen genügen vollständig; da wir sie nicht ungetrennt reizen können, so wird die Interferenz ihrer Wirkungen in Frage kommen, und es erscheint begreiflich, dass die secretorischen Fasern zunächst überwiegen; ist aber der secretorische Process schon annähernd maximal, so werden, namentlich bei starker Reizung, die hemmenden leicht in der Wirkung iberwiegen können, worin sogar eine gewisse Zweckmässigkeit liegen würde. Auch anf dem Gebiete der Gefässinnervation kennt man analoge Beobachtungen. Erst dann würde B i e d e r m a n n's Annahme entgegengesetzter Zellprocesse zur Nothwendigkeit werden, wenn die Nervenrejung eine negative Schwankung bis zur Um ke h r u $\mathrm{n}$ g des Ruhestroms bewirken würde.

Wenn ich auch die Biedermann'sche Heranziehung einer umgekehrten electromotorischen Veränderung an diesem Object als unnöthig und nicht völlig klar bezeichnen musste, bin ich doch weit entfernt, den zu Grunde liegenden Gedanken allgemein zurückzuweisen, zumal ich selbst der Erste war, der ihn ausgesprochen hat, was Biedermann's Darstellung, welche nur Hering als Urheber anfïhrt, nicht erkennen lässt. Grade der Anelectrotonus, den Biedermann als Ausgangspunkt dieser Idee anführt, hat mich schon vor 27 Jahren veranlasst, die Möglichkeit auszusprechen, dass die synthetische (assimilative, aufsteigende) Veränderung. der Nervensubstanz dieselbe positiv electrisch gegen nicht oder weniger veränderte macht ${ }^{1}$ ), ein Gedanke, der mir bitteren Hohn seitens $d u$ Bois-Reymond's ${ }^{2}$ ) eingetragen bat. Ich habe ihn,

1) Weitere Untersuchungen zur Physiologie der Muskeln und Nerven. Berlin 1867. s. 42.

2) Monatsberichte d. Berliner Acad. 1867. S. 628. (Gesammelte Ab. handlungen Bd. 2. S. 344.) 
obwohl ich ihn nie ans dem Auge verlor ${ }^{1}$ ), nur deshalb für den Electrotonus nicht weiter urgirt, weil ich ihn zur Erklärung desselben nicht nöthig fand, und auch jetzt noch, abweichend von Hering und Biedermann, nicht nöthig finde. Auch dass Biedermann wie eine Abweichung der Hering'schen Anschaung von der meinigen anführt, dass Hering die electromotorischen Wirkungen nicht auf Zustände, sondern auf Vorgänge in den erregbaren Substanzen zurückführt, kann ich nicht anerkennen ${ }^{2}$ ); auch ich habe stets nur von Vorgängen, zuerst von Spaltungsgeschwindigkeiten, später ron Absterben, Erstarren, Thätigkeit, Alteration als Ursache des electromotorischen Gegensatzes gesprochen; He ring's Theorie ist nicht Modification, sondern weitere Ausbildung der meinigen. Selbst für Hering's Hypothese, dass die assimilative Veränderung auch antagonistische Empfindungen hervorbringen kann, muss ich meine Priorität wahren ${ }^{3}$ ). Aber diese Idee ist von Hering in so vorzïglicher Weise an zahlreichen Thatsachen der Sinnesphysiologie gepruft und erprobt worden, dass ich ihm gern das Hauptverdienst zuerkenne, da es in unserer Wissenschaft wesentlich auf die untersuchende Arbeit ankommt.

Scbliesslich möchte ich auf einige von Biedermann nicht erwähnte Beobachtungen über Hautströme hinweisen, welche ich selbst und meine Mitarbeiter gemacht haben, nämlich das Vorkommen verkehrter Ruheströme an der Froschbaut ${ }^{4}$ ), den Einfluss der Temperatur auf die Hautströme ${ }^{5}$ ), endlich den Einfluss des Atropins und des Pilocarpins ${ }^{6}$ ).

Zusatz bei der Correctur. Soeben bemerke ich im Schlusspassus der Arbeit von Bayliss und Bradford (Journ. of physiol. Bd. 7. S. 229) die von mir auch im Jahresbericht pro 1886 ibersehene ganz kurze Notiz, dass diese Autoren am Laubfrosch (tree frog) die Schwankung "wie beim Frosch" gefunden haben; sie haben nur ein einziges Exemplar untersucht.

1) Vgl. u. A. dies Arehiv Bd. 31. S. 109.

2) Reid (Journ. of physiol. Bd. 16, S. 367) spricht Biedermann diesen Irrthum nach.

3) Weitere Untersuchungen etc. Berlin 1867. S. $52 \mathrm{f}$.

4) Dies Archiv Bd. 17. S. 302. Anm.

5) Dies Archiv Bd. 22. S. 31 f., Bd. 34. S. $422 \mathrm{f}$.

6) Dies Archiv Bd. 22. S. 34. 